ARTICLE

Received 23 May 2016 | Accepted 26 Aug 2016 | Published 7 Oct 2016

\title{
Mutually exclusive sense-antisense transcription at FLC facilitates environmentally induced gene repression
}

\author{
Stefanie Rosa ${ }^{1}$, Susan Duncan ${ }^{1} \&$ Caroline Dean ${ }^{1}$
}

\begin{abstract}
Antisense transcription through genic regions is pervasive in most genomes; however, its functional significance is still unclear. We are studying the role of antisense transcripts (COOLAIR) in the cold-induced, epigenetic silencing of Arabidopsis FLOWERING LOCUS C (FLC), a regulator of the transition to reproduction. Here we use single-molecule RNA FISH to address the mechanistic relationship of FLC and COOLAIR transcription at the cellular level. We demonstrate that while sense and antisense transcripts can co-occur in the same cell they are mutually exclusive at individual loci. Cold strongly upregulates COOLAIR transcription in an increased number of cells and through the mutually exclusive relationship facilitates shutdown of sense FLC transcription in cis. COOLAIR transcripts form dense clouds at each locus, acting to influence FLC transcription through changed H3K36me3 dynamics. These results may have general implications for other loci showing both sense and antisense transcription.
\end{abstract}

\footnotetext{
${ }^{1}$ Department of Cell and Developmental Biology, John Innes Centre, Norwich Research Park, Norwich NR4 7UH, UK. Correspondence and requests for materials should be addressed to C.D. (email: caroline.dean@jic.ac.uk).
} 
T here is considerable interest in understanding the mechanisms through which antisense transcription functions in gene regulation ${ }^{1,2}$. A classic example of environmentally mediated regulation involving antisense RNAs is vernalization, which in Arabidopsis involves the cold-induced epigenetic silencing of FLOWERING LOCUS C (FLC), a repressor of flowering ${ }^{3,4}$. An early phase in vernalization is the upregulation of a set of antisense transcripts, named COOLAIR that encompass the entire FLC locus ${ }^{5}$. Before cold COOLAIR transcription is repressed by the NDX1 homeodomain protein, which stabilizes an R-loop (where the nascent transcript invades the DNA helix) over the COOLAIR promoter ${ }^{6}$. The cold-induced COOLAIR transcription accelerates repression of FLC transcription ${ }^{7}$, but the mechanism through which this occurs is not known. As cold exposure continues, epigenetic silencing of FLC quantitatively increases, progressively reducing FLC re-activation when the plants return to warm conditions. This quantitative memory is due to a switch of epigenetic state at FLC in an increasing fraction of cells ${ }^{8}$. This switch is cell-autonomous, mediated by a modified Polycomb repressive complex 2 with the local chromatin environment of each locus instructing its own epigenetic inheritance ${ }^{9}$. This mechanism involves COOLAIRdependent switching of opposing histone modification states (H3K36me3 to H3K27me3) and spreading of Polycomb silencing across the locus to maintain long-term memory of the silenced state $^{5,8,10,11}$.

To elucidate the role of COOLAIR in this Polycomb switching mechanism we used single-molecule fluorescence in situ hybridization (smFISH) to detect individual sense and antisense RNAs. We show that COOLAIR transcription is anti-correlated with FLC transcription and unspliced COOLAIR forms dense clouds around the FLC locus in the early weeks of cold. The nature and timing of these events reveals the importance of COOLAIR to promote efficient FLC transcriptional repression before Polycomb silencing.

\section{Results}

smFISH for cellular analysis. We adapted a smFISH method ${ }^{12,13}$ to visualize and quantify single RNA transcripts in plant cells. The developmental structure and transparency of the Arabidopsis root made it an ideal tissue for this procedure. FLC messenger RNA (mRNA) transcripts showed a graded reduction in each cell as the duration of cold exposure increased (Fig. 1a-c). This was not a general cold-induced reduction, as levels of a constitutively expressed gene Protein Phosphatase 2A (PP2A) remained high after 6-weeks cold exposure (Supplementary Fig. 1). Expression determined from this approach corresponded well to quantitative reverse transcription-PCR measurements of FLC mRNA (Supplementary Fig. 2a).

In contrast to the graded and slow reduction in number of FLC mRNA molecules, FLC non-spliced transcripts (representing transcription) decreased quickly with many cells showing no signal after 2-weeks of cold (Fig. 1a,b,d). No obvious mRNA re-localization was revealed, as the mRNA signals showed no change in distribution before or after cold (Fig. 1b; Supplementary Fig. 2b). These observations suggest that dynamic molecular changes at the locus rapidly turn off FLC transcription in response to cold.

COOLAIR accumulates over FLC loci after cold exposure. Since COOLAIR is activated early during cold, we asked whether it has a role in this process. Intronic probes that detected non-spliced transcript (Fig. 2a) revealed signal in only $\sim 3 \%$ of total cells in warm-grown plants (Fig. 2b), explaining the overall low levels of COOLAIR expression ${ }^{5}$. However, after 2 -weeks of cold exposure, non-spliced COOLAIR increased in many cell types in the root, such that overall the number of cells expressing COOLAIR increased to $\sim 30 \%$ (Fig. $2 \mathrm{~b}$ ). In addition, COOLAIR foci increased in intensity and number in each nucleus (Fig. 2c,e; Supplementary Fig. 3). The distribution was reminiscent of nuclear bodies implicated in processing and maturation of ribonucleoprotein complexes and aberrant mRNAs ${ }^{14,15}$. Sequential smFISH-immunolabelling of both non-spliced COOLAIR and the Cajal body marker U2B' in the same cell, however, showed no co-localization (Supplementary Fig. 4). To understand the origin of the different foci we performed a sequential RNA-DNA FISH in cold-exposed root cells. This showed the large but not the small COOLAIR foci colocalized with FLC DNA FISH signals (Fig. 2d, Supplementary Fig. 5). The larger accumulations of COOLAIR are thus in 'clouds' covering each FLC locus. We have previously detected COOLAIRchromatin interactions using chromatin isolation by RNA purification ${ }^{7}$. However, these correspond to localized and tightly integrated structures such as the R-loop at the COOLAIR promoter' ${ }^{6}$. The COOLAIR 'clouds' detected by FISH appear to be a more general association of the COOLAIR non-spliced transcript with FLC chromatin. Both types of data support a cis-regulatory function for COOLAIR transcription/transcripts in FLC regulation. Double labelling with COOLAIR exonic and intronic probes revealed that the large COOLAIR clouds invariably labelled with both probe sets and thus represent non-spliced transcript at the locus (Supplementary Fig. 6a,c). In the large majority of cells the small foci showed intronic signals only, however, occasionally we also observed small foci labelling with exonic probes alone (Supplementary Fig. 6c). We speculate that these smaller foci might correspond mainly to intron 1 lariats dispersed throughout the nucleus before degradation. Probes to COOLAIR exonic sequences did not reveal signals in the cytoplasm (Supplementary Fig. 6b). We have previously found spliced COOLAIR enriched in cytoplasmic fractions ${ }^{7}$, so the absence of cytoplasmic signal in the FISH may be due to the extensive secondary structure of the spliced COOLAIR transcripts ${ }^{16}$. COOLAIR levels were higher in $n d x 1-1$, a mutant defective in the homeodomain protein that represses COOLAIR transcription through stabilization of the R-loop at the COOLAIR promoter ${ }^{6}$ supporting the view that cold and NDX1 regulate COOLAIR transcription independently (Supplementary Fig. 7).

To understand the basis of the accumulation of the COOLAIR clouds at the FLC locus, we generated probes specific for either the $5^{\prime}$ or $3^{\prime}$ end of the COOLAIR largest intron (Fig. 2f). In some cells the $5^{\prime}$ set gave a higher signal, whereas in others the $5^{\prime}$ and $3^{\prime}$ sets gave similar signals (Fig. 2g,h, Supplementary Fig. 8), which we interpret to indicate the clouds are likely to be formed from both increased transcription and transcript accumulation at the locus. More detailed analysis of COOLAIR transcription rate and turnover will be required to fully elucidate this. The co-localization of COOLAIR $5^{\prime}$ and $3^{\prime}$ probe set provides an important control for off-target signals validating the specificity of the probe sets used, similar to the two-colour approach used by Cabili et al. ${ }^{17}$. A similar control with FLC $5^{\prime}$ and $3^{\prime}$ intronic probes (Supplementary Fig. 2c) and co-localization in the DNA-RNA FISH confirmed the specificity of the smFISH probes (Supplementary Fig. 2d).

COOLAIR and FLC transcription are mutually exclusive. We then asked whether formation of COOLAIR clouds would affect $F L C$ transcription at the same locus. We undertook a thorough analysis of the accumulation of COOLAIR and FLC in single cells over a time course of cold exposure (Fig. 3a). Although FLC sense 
a
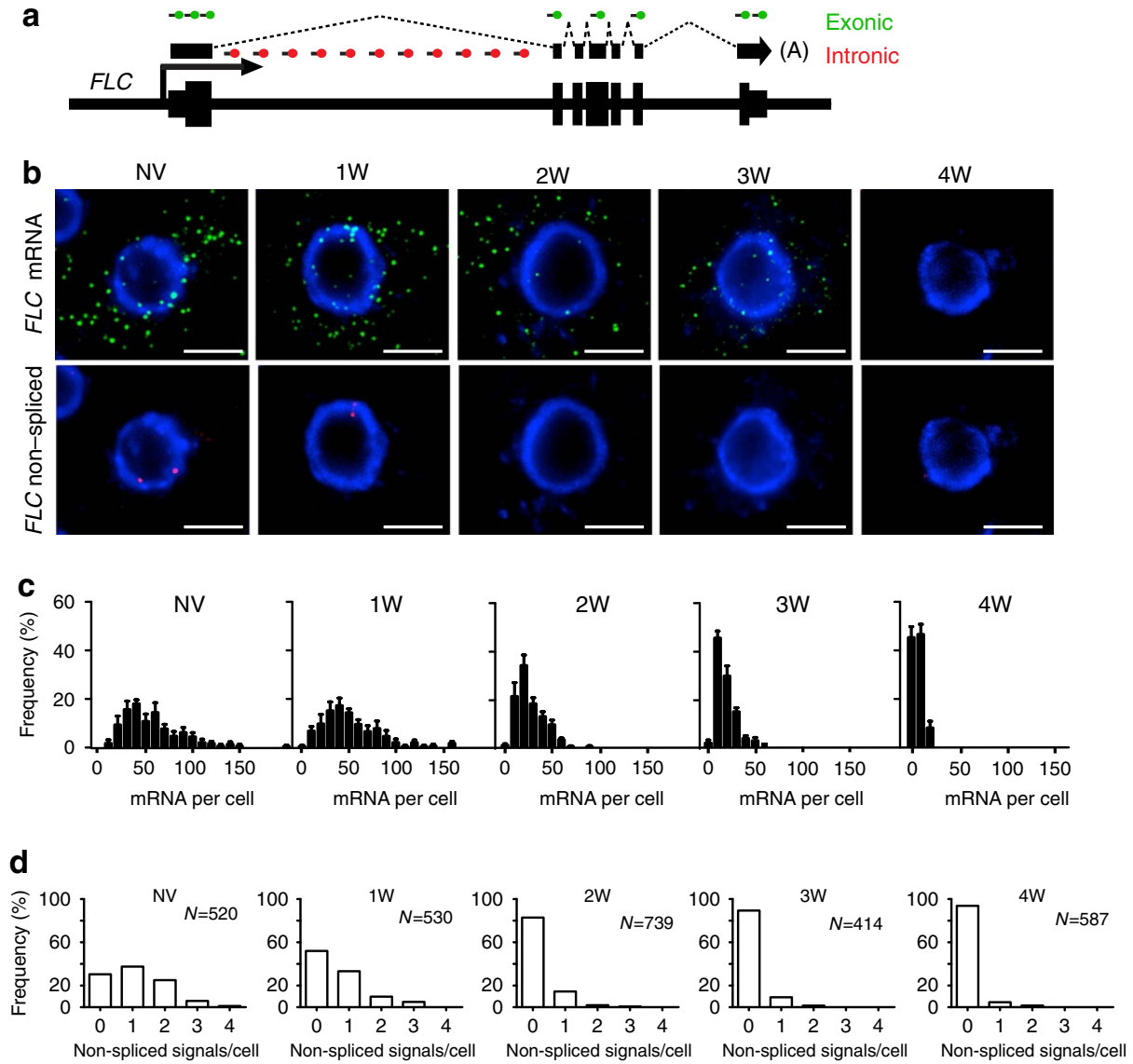

Figure 1 | Single-cell quantification of mRNA expression and $\boldsymbol{F L C}$ transcription during vernalization. (a) Schematic of the probes used to detect $F L C$ transcripts: intronic (red) and exonic (green). (b,c) Cytoplasmic FLC mRNA decreases gradually in the cold. (b) Representative images of cells showing the gradual decrease of FLC mRNA (green) and the reduction in nuclear non-spliced FLC transcripts (red) in the cold. DNA labelled with DAPI (blue). Scale bar, $5 \mu \mathrm{m}$. (c) Frequency distribution of FLC mRNA molecules per cell in non-vernalized plants (NV) and after different weeks of cold (1W, 2W, 3W, 4W). (d) Quantification of active sites of transcription as judged by non-spliced signal at the time points depicted in $\mathbf{c}$. $N=$ number of cells analysed. All error bars are $+/-$ s.e.m.

and antisense transcripts could co-occur in single cells (Fig. 3c), they were always transcribed from different alleles at any given time. Sense and antisense expression at individual loci were anti-correlated in both non-vernalized and cold-exposed cells (Fig. 3b), indicating transcription at the locus is unidirectional at any given time. This supports the view that COOLAIR is retained at the locus from which it is transcribed and acts in cis to regulate FLC. Analysis of FLC and COOLAIR expression in different genotypes had previously shown a positive correlation in expression ${ }^{5,18}$. The apparent contradiction between smFISH and the whole cell population data is likely due to mutually exclusive transcription of the sense or antisense strand both similarly influenced by trans factors and the local chromatin environment. Cold exposure would induce additional COOLAIR transcription at most loci, facilitating shutdown of FLC transcription and breaking the correlation.

To test this hypothesis, we asked whether this sense-antisense mutual exclusivity had implications for FLC transcription. We exploited the expression of COOLAIR enriched at vasculature precursor cells (Fig. 4a; Supplementary Fig. 9) and asked if the extent of FLC repression depended on the presence of COOLAIR. After 2-weeks of cold FLC expression had decreased $\sim 20 \%$ in non-COOLAIR expressing cells (Fig. 4c) and $\sim 75 \%$ in COOLAIR expressing cells (Fig. $4 \mathrm{~d}$ ). To confirm this difference was due to COOLAIR accumulation we analysed FLC transcriptional downregulation in a line $(F L C-T E X)^{7}$ defective in cold induction of COOLAIR (Fig. 4b). FLC sense transcription was less effectively downregulated in this line after 2-weeks of cold (Fig. 4d). Importantly, this effect was only observed in COOLAIR expressing cells, as no change in $F L C$ repression was observed in non-COOLAIR expressing cells (Fig. 4c). These data indicate that the accumulation of COOLAIR accelerates FLC repression during cold exposure.

\section{Discussion}

Our ability to analyse COOLAIR at the single cell level has revealed that an early step in vernalization is the accumulation of COOLAIR transcripts at the FLC locus. COOLAIR upregulation coincides with the disruption of a gene loop at $F L C^{19}$ and accelerates sense transcriptional shutdown within 1-2 weeks as compared with the 4 weeks required for maximal intragenic Polycomb nucleation $^{8}$-(Fig. 4e). The anti-correlation of COOLAIR and FLC transcription at the gene level would seem to exclude a cis-based colliding polymerase transcriptional interference mechanism. There is also no evidence for double-stranded RNA and the RNA interference machinery being required for vernalization ${ }^{20}$. We, therefore, propose that both the process of antisense transcription, and the presence of clouds of cis-tethered transcript, effectively repress FLC transcription. Indeed, disruption of COOLAIR predominantly affects the cold-induced reduction of $\mathrm{H} 3 \mathrm{~K} 36 \mathrm{me} 3$, the opposing chromatin mark to the 
a

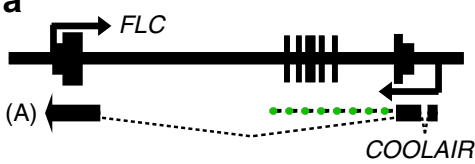

b
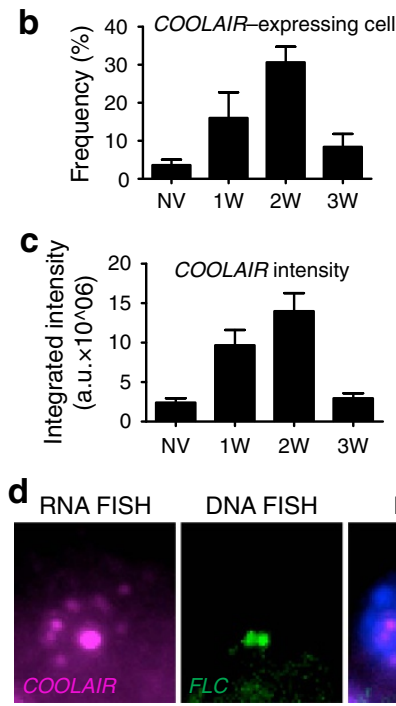

e

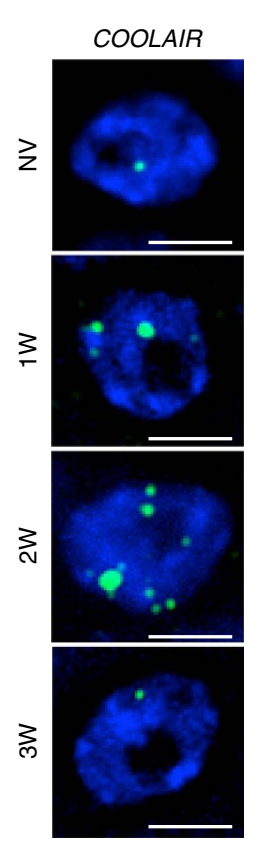

f
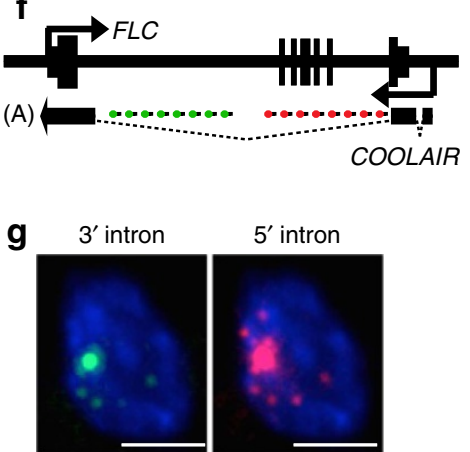

$5^{\prime}$ intron

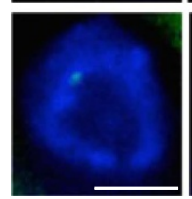

h

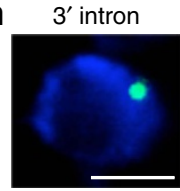

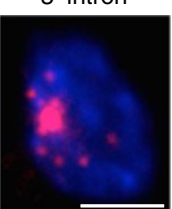

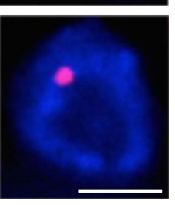

$5^{\prime}$ intron

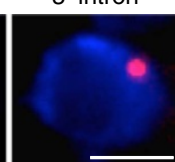

Figure 2 | COOLAIR transcription rapidly increases in some cells in the cold and coats the locus. (a) Schematic of the probes used to detect COOLAIR non-spliced transcripts. (b) Frequency of cells showing COOLAIR signals with increasing cold exposure (non-vernalized (NV), 1W, 2W, 3W). Error bars are $+/-$ s.e.m., with $N=250$ cells. (c) Quantification of total integrated intensity of the large COOLAIR foci at different time points with increasing cold exposure. Error bars are $+/$ - s.e.m., with $N=60$ cells. (d) Representative image of a nucleus showing co-localization of COOLAIR non-spliced RNA smFISH signals (magenta) with FLC DNA FISH signals (green). (e) Representative images of nuclei hybridized with COOLAIR smFISH probes (green), depicted in $\mathbf{a}$, in NV and plants exposed to different weeks of cold (1W, 2W, 3W). The data show the increase in the number and intensity of COOLAIR foci per nucleus. (f) Schematic of the probes used to detect the $5^{\prime}$ (red) and $3^{\prime}$ (green) end of COOLAIR largest intron. (g) Representative images of nuclei showing higher signals at the $5^{\prime}$-end, in plants exposed to 2 -weeks of cold. (h) Representative image of a nucleus showing equally high signals at the $5^{\prime}$ and $3^{\prime}$ end, in plants exposed to 2-weeks of cold. DNA labelled with DAPI (blue). Scale bar, $5 \mu \mathrm{m}$.

a

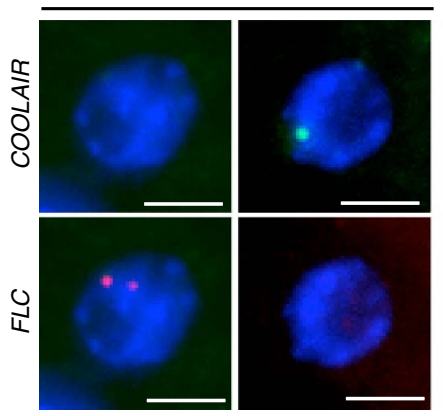

b

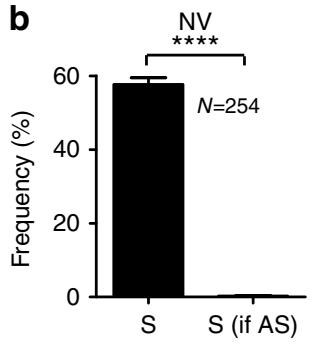

NV

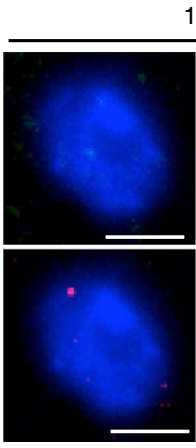

$1 \mathrm{~W}$
$1 \mathrm{~W}$

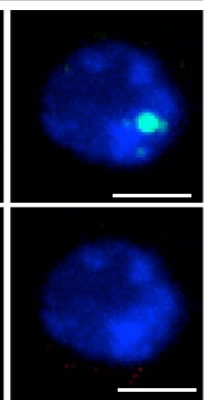

$2 \mathrm{~W}$

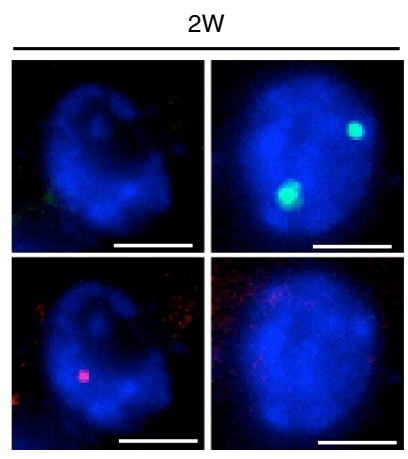

C

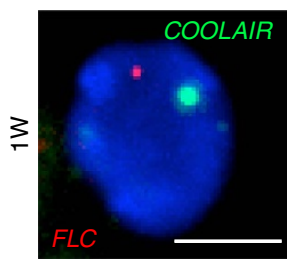

Figure 3 | FLC and COOLAIR transcription are anti-correlated at the level of the locus. (a) Representative images of nuclei hybridized with intronic smFISH probes for COOLAIR (green) and FLC (red) showing mutually exclusive transcription. Plants were given no cold (non-vernalized plants (NV)) or different weeks of cold (1W, 2W). DNA labelled with DAPI (blue). Scale bar, $5 \mu \mathrm{m}$. (b) Frequency of loci transcribing FLC, when COOLAIR is not present (S) or is detectable at the same locus (S if AS). FLC and COOLAIR transcription are anti-correlated at the level of the gene throughout all time points analysed $(\mathrm{NV}, 1 \mathrm{~W}, 2 \mathrm{~W}) . \mathrm{N}=$ number of cells analysed. All error bars are $+/-$ s.e.m. (c) Representative image of a nucleus hybridized with intronic smFISH probes against non-spliced COOLAIR (green) and FLC (red) in plants exposed to 1-week cold (1W), showing transcription of FLC and COOLAIR from different alleles in the same cell. Scale bar, $5 \mu \mathrm{m}$. 

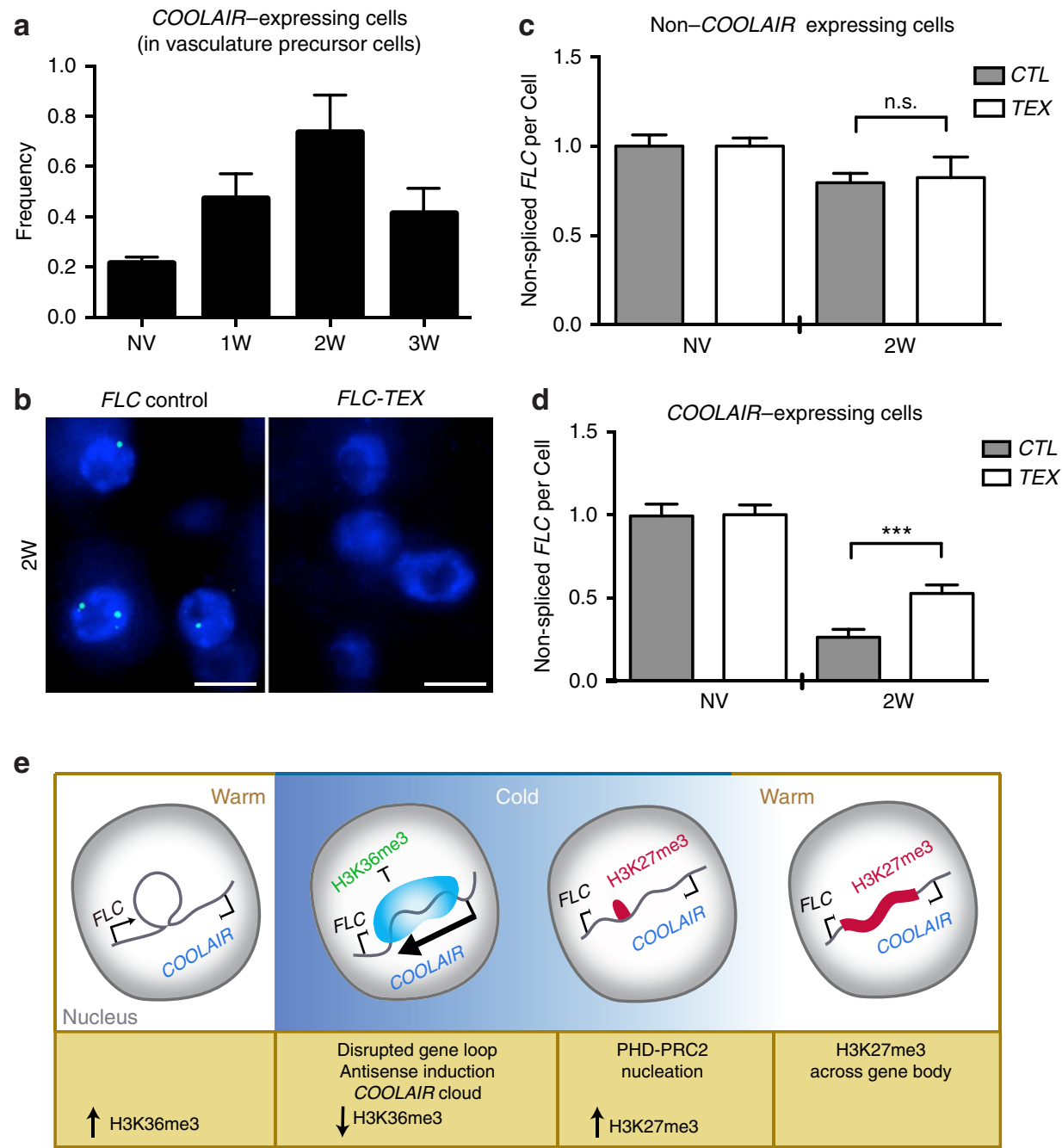

Figure 4 | COOLAIR facilitates shutdown of FLC sense expression. (a) Frequency of vasculature precursor cells showing COOLAIR transcription after increasing cold exposure (non-vernalized (NV), 1W, 2W, 3W). Error bars are $+/-$ s.e.m., with $N=200$ cells. (b) Representative images of nuclei hybridized with intronic smFISH probes against COOLAIR (green) in plants vernalized for 2 weeks (2W), in control transgenic plants carrying an intact FLC gene and plants carrying an FLC terminator exchange construct lacking COOLAIR promoter (FLC-TEX). Scale bar, $5 \mu \mathrm{m}$. (c) Quantification of non-spliced FLC transcripts per cell, in non-COOLAIR expressing cells from control transgenic plants (CTL) and a FLC-TEX line (TEX), in NV plants and plants vernalized for 2 weeks (2W); frequencies were normalized to NV values. Error bars are $+/-$ s.e.m., with $N=320$ cells. (Student's $t$-test, n.s., not-significant) (d) Quantification of non-spliced FLC transcripts per cell, in COOLAIR expressing cells from control transgenic plants (CTL) and a FLC-TEX line (TEX), in NV plants and plants vernalized for 2 weeks ( $2 \mathrm{~W}$ ); frequencies were normalized to NV values. Error bars are $+/-$ s.e.m., with $N=300$ cells (Student's $t$-test, $\left.{ }^{\star \star \star} P<0.001\right)$. (e) Schematic representing the current understanding of the role of COOLAIR in vernalization.

Polycomb-deposited $\mathrm{H} 3 \mathrm{~K} 27 \mathrm{me} 3$, at the nucleation region in $F L C^{7,11}$. COOLAIR transcription/transcripts could trigger a facultative heterochromatin state, potentially through blocking the activity/recruitment of $\mathrm{H} 3 \mathrm{~K} 36 \mathrm{me} 3$ modification complexes, or enhancing recruitment of $\mathrm{H} 3 \mathrm{~K} 36 \mathrm{me} 3$ demethylases, to maintain repression of FLC transcription as a necessary step to enable Polycomb-induced epigenetic switching ${ }^{21,22}$

Our analysis reveals that COOLAIR activity differs in many ways from well-characterized antisense transcripts in S. cerevisiae, such as from PHO84 antisense that is continuously transcribed at a low level with polyadenylated antisense transcripts rapidly exported to the cytoplasm ${ }^{23}$, or from the GAL10 locus where sense and antisense transcripts are transcribed simultaneously from the same locus ${ }^{24}$. Conversely, COOLAIR shows many parallels with the non-coding Xist RNA involved in mammalian $\mathrm{X}$-inactivation ${ }^{25}$; timed expression, cis-localization to a silenced allele and activity as a prelude to Polycomb silencing ${ }^{26}$. These parallels suggest that antagonistic sense-antisense transcription might be a core mechanism underlying the evolution of many epigenetic mechanisms.

\section{Methods}

Plant material and growth conditions. Seeds were stratified for 3 days at $5{ }^{\circ} \mathrm{C}$ before germination in a growth cabinet (Sanyo MLR-351H) in vertically oriented petri dishes containing Murashige and Skoog (MS) media minus glucose (16 h light, $\left.100 \mu \mathrm{mol} \mathrm{m}-2 \mathrm{~s}^{-1}, 22^{\circ} \mathrm{C} \pm 1^{\circ} \mathrm{C}\right)$. Seeds were surface sterilized in $5 \% \mathrm{v} / \mathrm{v}$ sodium hypochlorite for $5 \mathrm{~min}$ and rinsed three times in sterile distilled water.

The lines used in this study were described, previously: ColFRI (ref. 27), FLC-TEX $X^{7}, F L C$ transgenic ${ }^{7}, n d x 1-1 F R I$ (ref. 6).

Synthesis of the probes. The probes were chosen with the following criteria: 1 ) they should be roughly 17-22 bases long, 2) their GC content should be as close to $45 \%$ as possible and 3) there should at least three nucleotides of space between their target regions. We used the online program Stellaris Probe Designer version 2.0 from Biosearch Technologies. For probe sequences see Supplementary Tables $1-8$. 
smFISH procedure on root squashes. Seedlings were removed from the media and the root tips were cut using a razor blade and placed into glass wells containing $4 \%$ paraformaldehyde (PFA) and fixed for $30 \mathrm{~min}$. Roots were then removed from fixative and washed three times with nuclease free $1 \times$ PBS (Thermo Scientific, Lutterworth, UK). Several roots were then arranged on poly-L-lysine slide (Thermo Scientific, Lutterworth, UK) and covered by a glass cover slip (Slaughter, Uppminster, UK). The meristems were then squashed by tapping the cover slips, and immediately after each slide was submerged (together with the cover slip) for a few seconds in liquid nitrogen until frozen. The cover slips were then flipped off the slides using a razor blade and the roots were left to dry at room temperature for $30 \mathrm{~min}$.

Tissue permeabilization was achieved by immersing the samples in $70 \%$ ethanol for a minimum of $1 \mathrm{~h}$. The ethanol was then left to evaporate at room temperature for $5 \mathrm{~min}$ before two washes were carried out with wash buffer (containing 10\% formamide and $2 \times$ SSC). One-hundred microlitres of hybridization solution (containing $10 \%$ dextran sulfate, $2 \times$ SSC and $10 \%$ formamide) with each probe set at a final concentration of $250 \mathrm{nM}$ was then added to each slide. Cover slips (Slaughter, Uppminster, UK) were carefully laid over the samples to prevent the evaporation of the buffer and the probes were left to hybridize at $37^{\circ} \mathrm{C}$ overnight in the dark.

The hybridization solution containing unbound probes was pipetted off in the morning. Each sample was washed twice with wash buffer with the second wash left to incubate for $30 \mathrm{~min}$ at $37^{\circ} \mathrm{C}$. One-hundred microlitres of the nuclear stain 4,6-diamidino-2-phenylindole (DAPI; $100 \mathrm{ng} \mathrm{ml}^{-1}$ ) was then added to each slide and left to incubate at $37^{\circ} \mathrm{C}$ for $30 \mathrm{~min}$. The DAPI was removed and $100 \mu \mathrm{l}$ $2 \times$ SSC was added and then removed. One-hundred microlitres GLOX buffer minus enzymes $(0.4 \%$ glucose in $10 \mathrm{mM}$ Tris, $2 \times$ SSC) was added to the samples and left to equilibrate for $2 \mathrm{~min}$. This was removed and replaced with $100 \mu \mathrm{l}$ of GLOX buffer containing enzymes (glucose oxidase and catalase). Glucose oxidase (\#G0543 from Sigma) and catalase (\#C3155 from Sigma), $1 \mu$ of each enzyme is added to a total of $100 \mu \mathrm{l}$ of GLOX minus enzymes. The samples were then covered by $22 \times 22 \mathrm{~mm}$ No.1 cover slips (Slaughter, Uppminster, UK), sealed with nail varnish and immediately imaged.

Combined smFISH with immunofluorescence. We performed a sequential smFISH-immunofluorescence by first performing the RNA FISH using smFISH probes using the protocol outlined above and then performing the immunofluorescence. For sequential immunofluorescence-smFISH, cover slips were unmounted after imaging RNA FISH and washed three times in $4 \times-\mathrm{SSC} / 0.2 \%$ Tween at $37^{\circ} \mathrm{C}$. After slides were re-fixed with $4 \%(\mathrm{w} / \mathrm{v})$ formaldehyde freshly made from PFA in PBS buffer for 10 min. After washing in PBS for $5 \mathrm{~min}$, the slides were digested in a mixture of $1 \%$ dolicalase, $0.5 \%$ cellulase, $0.025 \%$ pectolyase) at room temperature for $10 \mathrm{~min}$. After enzyme treatment, roots were washed in PBS three times for 5 min each. Then the slides were blocked with $4 \%$ BSA in PBS for 50 min, followed by incubation with primary antibody. AntiU2B' antibody from mouse (1:20) (gift from Peter Shaw ${ }^{28}$ ) was used as a primary antibody in $1 \%$ BSA in PBS. The slides were incubated at $37^{\circ} \mathrm{C}$ for $1 \mathrm{~h}$ in a humid box. After washing the slides in PBS for 5 min each, anti-mouse IgG A488 conjugate (Invitrogen) was applied as secondary antibody (1:200) and slides were incubated for $1 \mathrm{~h}$ in a humid dark box at $37^{\circ} \mathrm{C}$. After washing the slides three times in PBS for $5 \mathrm{~min}$ each, the specimens were counter- stained with $1 \mathrm{~g} \mathrm{ml}^{-1}$ DAPI (49,6-diamidino-2-phenylindole dihydrochloride hydrate; Sigma) and mounted in Vectashield (Vector Lab). In order to find the cells previously imaged for smFISH, we saved the stage positions of those cells at the microscope and acquired large image tiles in order facilitate the identification of the cells.

Combined RNA-DNA FISH. For sequential RNA-DNA FISH, cover slips were unmounted after imaging RNA by smFISH (using the protocol outlined above) and washed three times in $4 \times-\mathrm{SSC} / 0.2 \%$ Tween at $37^{\circ} \mathrm{C}$. After that slides were re-fixed with $4 \%(\mathrm{w} / \mathrm{v})$ formaldehyde freshly made from PFA in $1 \times$ PBS buffer for $10 \mathrm{~min}$ and washed again several times in $1 \times$ PBS. Slides were treated with $100 \mu \mathrm{g} \mathrm{ml}^{-1}$ RNase for $1 \mathrm{~h}$ at $37^{\circ} \mathrm{C}$ and washed twice in $1 \times$ PBS. After washing slides were digested in a mixture of $1 \%$ driselase, $0.5 \%$ cellulase, and $0.025 \%$ pectolyase for $10 \mathrm{~min}$ at $37^{\circ} \mathrm{C}$. Slides were then washed and re-fixed with $4 \%(\mathrm{w} / \mathrm{v})$ formaldehyde freshly made from PFA in PBS buffer for $10 \mathrm{~min}$. Slides were then transferred to a series of ethanol steps increasing to 70,85 and $100 \%$.

Probes were labelled with Digoxigenin-11-dUTP (\#11745816910, Roche) by nick translation. Bacterial artificial chromosome clone JAtY71K18, which contains an insert of $75 \mathrm{~kb}$, was used as a probe. The hybridization mixture $\left(20 \mathrm{ng} \mathrm{ml}^{-1}\right.$ labelled DNA, $50 \%$ formamide, $10 \%$ dextran sulfate, $2 \times$ SSC, $1 \mathrm{mg} \mathrm{ml}^{-1}$ salmon sperm (D9156, Sigma)) was denatured at $85^{\circ} \mathrm{C}$ for $10 \mathrm{~min}$ and applied to the slides. Slides containing the hybridization mixture were denatured for $7 \mathrm{~min}$ at $75^{\circ} \mathrm{C}$ (in a omnislide), and hybridized overnight at $37^{\circ} \mathrm{C}$. After hybridization, slides were washed at $42^{\circ} \mathrm{C}$ once in $2 \times$ SSC, twice in $20 \%$ formamide plus $0.1 \times$ SSC and twice in $2 \times$ SSC, and then twice in $2 \times$ SSC at room temperature and twice in $4 \times$ SSC plus $0.2 \%$ Tween-20. Then the slides were blocked in TNB $(0.1 \mathrm{M}$ TrisHCl, $0.15 \mathrm{M} \mathrm{NaCl}, 3 \% \mathrm{BSA}$ ) for $30 \mathrm{~min}$ at $37^{\circ} \mathrm{C}$. Digoxigenin-2́-deoxyuridine, 5 triphosphate probes were detected with anti-DIG-fluorescein antibody (\#11207741910, Roche) prepared in TNB buffer (1:100). Nuclei were counterstained with $1 \mathrm{mg} \mathrm{ml}^{-1}$ DAPI, and slides were mounted in Vectashield
(Vector Laboratories). In order to find the cells previously imaged for smFISH, we saved the stage positions of those cells at the microscope and acquired large image tiles in order facilitate the identification of the cells.

Image acquisition. A Zeiss Elyra PS1 inverted microscope was used for imaging. A $\times 100$ oil-immersion objective (1.46 NA) and cooled electron multiplying-CCD (charge-coupled device) Andor iXon 897 camera $(512 \times 512$ QE $>90 \%)$ was used to obtain all images in the standard, rather than super-resolution mode. The following wavelengths were used for fluorescence detection: for probes labelled with Quasar570 an excitation line of $561 \mathrm{~nm}$ was used and signal was detected at 570-640 nm; for probes labelled with Quasar670 an excitation line of $642 \mathrm{~nm}$ and signal was detected at $655-710 \mathrm{~nm}$; for DAPI an excitation line of $405 \mathrm{~nm}$ and signal was detected at wavelengths of $420-480 \mathrm{~nm}$. For all experiments series of optical sections with z-steps of $0.2 \mu \mathrm{m}$ were collected.

Z-stacks were deconvolved using AutoQuant X2 (Media Cybernetics). Maximum projections and analysis of three-dimensional pictures were performed using Fiji (an implementation of ImageJ, a public domain program by W. Rasband available from http://rsb.info.nih.gov/ij/).

Image analysis. The analysis of smFISH images consisted of two componentssegmentation and mRNA counting ${ }^{13}$. These two components were combined into an overall workflow that resulted in an image where each cell was annotated with the number of probes located in it. The image analysis workflow operated on image collections where each image represented a unique channel/z-stack pair. To separate the captured microscopy image into individual channel/z-stack pairs Bioformats was used, and the pipeline was implemented in the Python programming language.

Quantification of $\mathbf{3}^{\prime} / \mathbf{5}^{\prime}$ ratio for COOLAIR largest intron. Images were acquired with the same laser intensity; exposure time and gain for the two probe sets ( $3^{\prime}$ probes were labelled with Quasar 670 and $5^{\prime}$ probes with Quasar 570-see Image Acquisition for specific lasers and filter sets used). Intensity of intronic signals was calculated from maximum intensity projection images, as total integrated intensity of each dot after background subtraction.

Data availability. The data that support the findings of this study are available from the corresponding author upon request.

\section{References}

1. Clark, M. B. et al. The reality of pervasive transcription. PLoS Biol. 9, e1000625 (2011).

2. Pelechano, V. \& Steinmetz, L. M. Gene regulation by antisense transcription. Nat. Rev. Genet. 14, 880-893 (2013).

3. Michaels, S. D. \& Amasino, R. M. FLOWERING LOCUS C encodes a novel MADS domain protein that acts as a repressor of flowering. Plant Cell 11, 949-956 (1999).

4. Sheldon, C. C. et al. The FLF MADS box gene: a repressor of flowering in Arabidopsis regulated by vernalization and methylation. Plant Cell 11,445-458 (1999).

5. Swiezewski, S., Liu, F., Magusin, A. \& Dean, C. Cold-induced silencing by long antisense transcripts of an Arabidopsis Polycomb target. Nature 462, 799-802 (2009).

6. Sun, Q., Csorba, T., Skourti-Stathaki, K., Proudfoot, N. J. \& Dean, C. R-loop stabilization represses antisense transcription at the Arabidopsis FLC locus. Science 340, 619-621 (2013).

7. Csorba, T., Questa, J. I., Sun, Q. \& Dean, C. Antisense COOLAIR mediates the coordinated switching of chromatin states at FLC during vernalization. Proc. Natl Acad. Sci. USA 111, 16160-16165 (2014).

8. Angel, A., Song, J., Dean, C. \& Howard, M. A Polycomb-based switch underlying quantitative epigenetic memory. Nature 476, 105-108 (2011)

9. Berry, S., Hartley, M., Olsson, T. S., Dean, C. \& Howard, M. Local chromatin environment of a Polycomb target gene instructs its own epigenetic inheritance. elife 4, e07205 (2015).

10. De Lucia, F., Crevillen, P., Jones, A. M., Greb, T. \& Dean, C. A PHD-polycomb repressive complex 2 triggers the epigenetic silencing of $F L C$ during vernalization. Proc. Natl Acad. Sci. USA 105, 16831-16836 (2008).

11. Yang, H., Howard, M. \& Dean, C. Antagonistic roles for H3K36me3 and H3K27me3 in the cold-induced epigenetic switch at Arabidopsis FLC. Curr. Biol. 24, 1793-1797 (2014).

12. Raj, A., van den Bogaard, P., Rifkin, S. A., van Oudenaarden, A. \& Tyagi, S. Imaging individual mRNA molecules using multiple singly labeled probes. Nat. Methods 5, 877-879 (2008).

13. Duncan, S., Olsson, T., Hartley, M., Dean, C. \& Rosa, S. A method for detecting single mRNA molecules in Arabidopsis thaliana. Plant Methods 12, 13 (2016).

14. Cioce, M. \& Lamond, A. I. Cajal bodies: a long history of discovery. Annu. Rev. Cell Dev. Biol. 21, 105-131 (2005). 
15. Shaw, P. J. \& Brown, J. W. Plant nuclear bodies. Curr. Opin. Plant Biol. 7, 614-620 (2004).

16. Hawkes, E. J. et al. COOLAIR antisense RNAs form evolutionarily conserved elaborate secondary structures. Cell Rep. 16, 3087-3096 (2016).

17. Cabili, M. N. et al. Localization and abundance analysis of human lncRNAs at single-cell and single-molecule resolution. Genome Biol. 16, 20 (2015).

18. Liu, F., Marquardt, S., Lister, C., Swiezewski, S. \& Dean, C. Targeted 3' processing of antisense transcripts triggers Arabidopsis FLC chromatin silencing. Science 327, 94-97 (2010).

19. Crevillen, P., Sonmez, C., Wu, Z. \& Dean, C. A gene loop containing the floral repressor $F L C$ is disrupted in the early phase of vernalization. EMBO J. 32, 140-148 (2013).

20. Schmitz, R. J., Hong, L., Fitzpatrick, K. E. \& Amasino, R. M. DICER-LIKE 1 and DICER-LIKE 3 redundantly act to promote flowering via repression of FLOWERING LOCUS C in Arabidopsis thaliana. Genetics 176, 1359-1362 (2007).

21. Li, N., Joska, T. M., Ruesch, C. E., Coster, S. J. \& Belden, W. J. The frequency natural antisense transcript first promotes, then represses, frequency gene expression via facultative heterochromatin. Proc. Natl Acad. Sci. USA 112, 4357-4362 (2015).

22. Wu, Z. et al. Quantitative regulation of $F L C$ via coordinated transcriptional initiation and elongation. Proc. Natl Acad. Sci. USA 113, 218-223 (2016).

23. Castelnuovo, M. et al. Bimodal expression of PHO84 is modulated by early termination of antisense transcription. Nat. Struct. Mol. Biol. 20, 851-858 (2013).

24. Lenstra, T. L., Coulon, A., Chow, C. C. \& Larson, D. R. Single-molecule imaging reveals a switch between spurious and functional ncRNA transcription. Mol. Cell 60, 597-610 (2015).

25. Gendrel, A. V. \& Heard, E. Noncoding RNAs and epigenetic mechanisms during X-chromosome inactivation. Annu. Rev. Cell Dev. Biol. 30, 561-580 (2014).

26. Deuve, J. L., Bonnet-Garnier, A., Beaujean, N., Avner, P. \& Morey, C. Antagonist Xist and Tsix co-transcription during mouse oogenesis and maternal Xist expression during pre-implantation development calls into question the nature of the maternal imprint on the $\mathrm{X}$ chromosome. Epigenetics 10, 931-942 (2015).

27. Lee, I., Michaels, S. D., Masshardt, A. S. \& Amasino, R. M. The late-flowering phenotype of FRIGIDA and mutations in LUMINIDEPENDENS is suppressed in the Landsberg erecta strain of Arabidopsis. Plant J. 6, 903-909 (1994).
28. Boudonck, K., Dolan, L. \& Shaw, P. J. Coiled body numbers in the Arabidopsis root epidermis are regulated by cell type, developmental stage and cell cycle parameters. J. Cell Sci. 111, 3687-3694 (1998).

\section{Acknowledgements}

We thank Zhe Wu and Silvia Costa for comments on the manuscript, all members of the Dean and Howard groups for discussions and Mathew Hartley and Tjelvar Olsson for the help with the image analysis. This work was supported by the UK Biotechnology and Biological Sciences Research Council (BBSRC) grant BB/K00008X/1 and the Earth and Life Systems Alliance (a collaborative venture between John Innes Centre and University of East Anglia). C.D. acknowledges support from European Research Council Advanced grant MEXTIM and BBSRC Institute Strategic Programme grant BB/J004588/1.

\section{Author contributions}

S.R., S.D. and C.D. conceived the study and designed the experiments; S.R. and S.D. performed the experiments; S.R. and C.D. and wrote the manuscript.

\section{Additional information}

Supplementary Information accompanies this paper at http://www.nature.com/ naturecommunications

Competing financial interests: The authors declare no competing financial interests.

Reprints and permission information is available online at http://npg.nature.com/ reprintsandpermissions/

How to cite this article: Rosa, S. et al. Mutually exclusive sense-antisense transcription at FLC facilitates environmentally induced gene repression. Nat. Commun. 7, 13031 doi: 10.1038/ncomms13031 (2016).

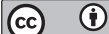

This work is licensed under a Creative Commons Attribution 4.0 International License. The images or other third party material in this article are included in the article's Creative Commons license, unless indicated otherwise in the credit line; if the material is not included under the Creative Commons license, users will need to obtain permission from the license holder to reproduce the material. To view a copy of this license, visit http://creativecommons.org/licenses/by/4.0/

(C) The Author(s) 2016 